\title{
Adaptive Cooperative Awareness Messaging for Enhanced Overtaking Assistance on Rural Roads
}

\author{
Annette Böhm, Magnus Jonsson and Elisabeth Uhlemann \\ CERES (Centre for Research on Embedded Systems) \\ Halmstad University, Halmstad, Sweden \\ \{Annette.Bohm, Magnus.Jonsson, Elisabeth.Uhlemann\}@hh.se
}

\begin{abstract}
Cooperative traffic safety applications such as lane change or overtaking assistance have the potential to reduce the number of road fatalities. Many emerging traffic safety applications are based on IEEE 802.11p and periodic position messages, so-called cooperative awareness messages (CAM) being broadcasted by all vehicles. In Europe, ETSI defines a periodic report rate of $2 \mathrm{~Hz}$ for CAMs. Although a high report rate is the key to early hazard detection, the $2 \mathrm{~Hz}$ rate has been chosen to avoid congestion in settings where the vehicle density is high, e.g., on major highways and in urban scenarios. However, on rural roads with a limited number of communicating vehicles, a report rate of $2 \mathrm{~Hz}$ leads to unnecessary delay in cooperative awareness. By adapting the CAM report rate depending on the specific application and road traffic density, and by making use of the priority levels provided by the 802.11p quality of service mechanism, we show that hazards can be detected earlier and the available bandwidth is used more efficiently, while not overexploiting the network resources.
\end{abstract}

\section{INTRODUCTION}

The introduction of Intelligent Transport Systems (ITS) relying on wireless communications promises to considerably improve the level of safety, comfort and efficiency on our roads. Cooperative ITS safety applications such as lane change assistance, highway entrance merging assistance and overtaking assistance have the potential to reduce the number of accidents and ultimately save lives. Many emerging traffic safety applications rely on the periodic exchange of status messages between vehicles through short to medium range vehicle-to-vehicle (V2V) or vehicle-to-roadside (V2R) communication. This cooperative view of the current traffic situation can then be used by various applications and proper actions can be taken (e.g., warnings can be issued to the driver or application specific warning messages can be sent to drivers of other vehicles). This requires a communication technology with low delay that allows broadcasting of messages between vehicles in the local surroundings.

The recent allocation of frequency in the US $(75 \mathrm{MHz}$ at the $5.9 \mathrm{GHz}$ band dedicated for ITS) and the introduction of IEEE $802.11 p$ [1], have paved the way for future implementations of proactive, cooperative ITS safety applications both in the US and worldwide. The physical and Medium Access Control (MAC) layers of IEEE 802.11p are an adaptation of the IEEE 802.11 Wireless Local Area Network (WLAN) standard including support for four Quality of Service (QoS)

This work was funded in part by the Knowledge Foundation, www.kks.se and the Swedish Transport Administration (Trafikverket) in participation with Volvo Technology. E. Uhlemann is partly funded by the Swedish Governmental Agency for Innovation Systems, Vinnova, through the VINNMER program, www.vinnova.se classes. The European Telecommunications Standards Institute (ETSI) has standardized a profile of IEEE $802.11 \mathrm{p}$ adapted to the $30 \mathrm{MHz}$ frequency spectrum at the $5.9 \mathrm{GHz}$ band allocated in Europe [2]. ETSI considers two types of messages, Cooperative Awareness Messages (CAM) [3] and Decentralized Environmental Notification Messages (DENM) [4]. CAMs are broadcasted periodically, stating vehicle speed, position and driving direction, whereas DENMs are event-driven and application specific warning messages. For Europe, ETSI defines a packet size of 800 byte and a periodic report rate of $2 \mathrm{~Hz}$ for CAMs.

Cooperative ITS applications are typically considered in settings where the vehicle density is high, such as on major highways and in urban scenarios, since cooperation between local vehicles can improve traffic efficiency and thereby also traffic safety. Sparsely trafficked rural roads are studied less frequently, although, due to their high relative speed limits and lack of physical counter measures (e.g. lack of guardrails), they account for a majority of the fatal traffic accidents today [5]. Although the CAM report rate is the key to early hazard detection, it has been limited to $2 \mathrm{~Hz}$ in order not to congest the network in scenarios with high vehicle densities. However, on rural roads with their limited number of communicating vehicles, a report rate of $2 \mathrm{~Hz}$ leads to a large amount of unallocated bandwidth and unnecessary delay in vehicle detection. The radio conditions along a rural road are highly influenced by environmental factors like topography or vegetation and a transmission range of $500 \mathrm{~m}$ at free line of sight (LOS) can suddenly drop to under $100 \mathrm{~m}$ [6], implying challenging timing requirements. By adapting the CAM report rate depending on the overall road traffic density and the specific application in use and by using the priority levels provided by the 802.11p QoS mechanism, we show that the available bandwidth can be used more efficiently and the hazard detection delay is reduced.

Several studies consider the CAM report rate (a.k.a. beacon rate) in vehicular networks. In [7], Vinel et al. suggest an analytical method to study the successful beacon delivery and mean beacon transmission delay. This approach does not consider the assignment of different roles and report rates to individual vehicles to improve the performance of specific applications. The focus of [8] lies on adapting the report rate based on vehicle speed and density and the position accuracy required by the application, but it does not, however, evaluate or propose methods to deal with application specific delay requirements. In [9], Sepulcre et al. study the effect of application-based report rate and transmission power on the channel busy time in a lane change warning application. In this paper, we focus on a cooperative over- 


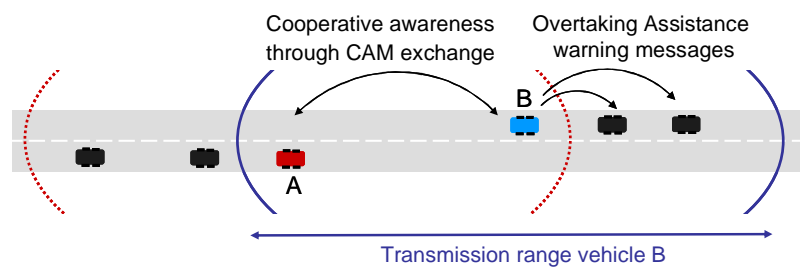

Transmission range vehicle $\mathrm{A}$

Figure 1: Overtaking Assistance scenario.

taking assistance application for rural roads with low vehicle density but high timing requirements. Through computer simulations, we evaluate whether different CAM report rates and QoS prioritization of messages can reduce the detection delay of on-coming traffic and thereby increase the time available for the driver to react and prevent a potential accident. We have taken special care to ensure that our solution does not require any alterations of the standard, but rather uses the available opportunities of tuning key parameters depending on the application requirements. Further, our simulator uses the results from real field measurements with 802.11p-enabled vehicles to model packet error probabilities. The rest of the paper is organized as follows: Section II describes the application Overtaking Assistance in more detail, while Section III presents our approach. The system model and the simulator are described in Sections IV and V respectively. The simulation results are presented and discussed in Section VI before Section VII concludes the paper.

\section{OVERTAKING ASSISTANCE}

Trucks or caravans queuing up traffic on single-lane rural roads are a common sight. Often on-coming traffic is detected too late to prevent dangerous overtaking maneuvers. This is a scenario where an IEEE 802.11p-based cooperative Overtaking Assistance application offers great potential to issue early warnings to drivers, prevent severe accidents and potentially save lives. As soon as a vehicle has detected oncoming traffic, most likely it is the vehicle leading the queue as it has the best opportunity to achieve LOS communications with the on-coming traffic, a specific overtaking warning message can be broadcasted to all vehicles within its transmission range, Figure 1. To detect on-coming traffic, periodic CAMs containing position, speed and direction are broadcasted by all vehicles, but the actual overtaking warning messages could alternatively be of DENM type.

We consider the following specifications: An overtaking warning, issued locally to the driver of the vehicle or broadcasted as a DENM, can only be viewed as successful if it reaches the relevant driver in time to react properly. We assume a driver reaction time of $2 \mathrm{~s}$ (which is realistic given a sudden and unexpected warning), but we do not add time for braking, as the warning should only prevent the driver from starting an overtaking maneuver or, in case he/she has already started it, to aboard the maneuver - not to bring the vehicle to a standstill. Once the on-coming traffic is detected, this information has to be spread to other vehicles in the vicinity and warnings to the corresponding drivers have to be issued. This adds additional time to the time span between the reception of the first CAM from an on-coming vehicle to a successful accident prevention. The $2 \mathrm{~s}$ reaction time can thus be seen as a deadline before which contact has to be established between the two driving directions, if the application should be considered useful. We therefore evaluate the performance of the Overtaking Assistance application based on how long before the $2 \mathrm{~s}$ reaction time contact is established between the two driving directions. Both a one sided connection and a full connection (both driving directions are aware of each other) are considered.

\section{Adaptive Cooperative Awareness Messaging}

As we base our application on the IEEE 802.11p standard, we first review its functionality. The MAC method in IEEE 802.11p is based on the Distributed Coordination Function (DCF) first introduced in the IEEE 802.11 standard. It uses Carrier Sense Multiple Access (CSMA), a random access MAC scheme where nodes compete with each other for access to the common medium. A node that has data to send listens to the medium a predefined time, called the interframe spacing (IFS), and is only allowed to send its data packet if the channel was determined to be free during this entire time period. Any detected activity on the channel during the IFS implies that a backoff procedure must be performed before a new transmission attempt is allowed. The backoff time is based on a random integer which is selected from a predefined contention window. For each successful transmission, i.e., successfully acknowledged by the intended recipient, the contention window is reduced, while its size is doubled (up to a predefined maximum) for each failed channel access attempt or unsuccessful transmission. Due to the broadcast nature of CAMs, acknowledge messages cannot be used (each packet has a varying set of indented receivers), and therefore only one backoff takes place, implying that the initial size of the contention window (CWmin) is maintained. Further, 802.11p uses Enhanced Distributed Channel Access (EDCA) with QoS support, first introduced in IEEE 802.11e. The size of the contention window, CWmin, and the size of the IFS, denoted Arbitration Interframe Space (AIFS) in EDCA, are used to provide support for four different QoS levels, Table 1. The higher the priority (i.e., the lower the access class), the smaller is the contention window, the smaller are its CWmin and CWmax values, and the shorter is the AIFS. Thereby, the probability of early channel access is increased for traffic classes with high priority. Despite this QoS differentiation, packet collisions within or between access classes are still possible and therefore, no timing guarantees can be given.

TABLE 1: EDCA PARAMETERS

\begin{tabular}{|c|c|c|c|}
\hline QoS class & CWmin & CWmax & AIFS \\
\hline $\mathbf{4}$ & 15 & 1023 & 9 \\
\hline $\mathbf{3}$ & 7 & 15 & 6 \\
\hline $\mathbf{2}$ & 3 & 7 & 3 \\
\hline $\mathbf{1}$ & 3 & 7 & 2 \\
\hline
\end{tabular}

In order to speed up the distribution of warning messages and use the bandwidth available on sparsely trafficked roads more efficiently, we propose a simple prioritization mechanism based on adapting the CAM report rate to the specific role the individual vehicle has in the application at hand. We further use the different QoS levels of the EDCA to distinguish between vehicles with different roles. In the Overtaking Assistance scenario studied in our simulations, we dis- 


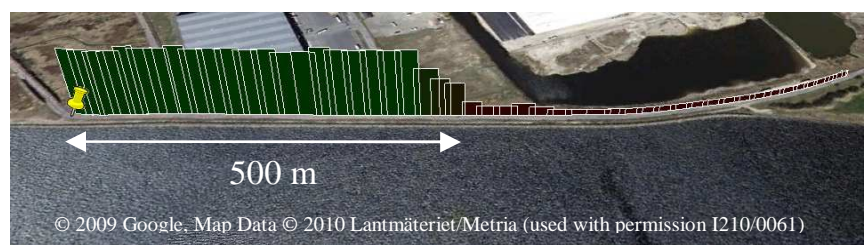

Figure 2: Variation of connectivity over distance at free LOS. Stationary sending vehicle (yellow pin), moving receiving vehicle.

tinguish between two such roles: "leading vehicles" (the first vehicle in a queue) and "regular vehicles" (all other vehicles in the queue). Note that a "queue" may contain only one vehicle. The two roles are classified as follows:

\section{A leading vehicle:}

- is defined as a vehicle at a distance larger than the transmission range from the vehicle in front.

- uses the parameters of the highest priority data traffic class provided by $802.11 \mathrm{p}$, QoS class 1 .

- uses a higher CAM report rate (e.g. 10 or $20 \mathrm{~Hz}$ ) than regular vehicles.

\section{A regular vehicle:}

- is defined as a vehicle at a distance less than the transmission range from the vehicle in front.

- uses the parameters of the lowest priority data traffic class provided by $802.11 \mathrm{p}$, QoS class 4 .

- sends its packets with a lower report rate than leading vehicles (e.g. $2 \mathrm{~Hz}$ as suggested by the standard).

Since the $2 \mathrm{~Hz}$ CAM update frequency used in the standard was chosen with densely trafficked highway and urban scenarios in mind, the available bandwidth is not efficiently used by the relatively low number of communicating vehicles on a rural road. This margin of unused bandwidth can be exploited by increasing the report rate of prioritized vehicles (in our case, a leading vehicle). In the case of the application Overtaking Assistance, a high report rate, i.e. an increased number of transmission attempts per time unit, increases the probability of early detection of on-coming traffic. There is, however, a tradeoff between an increased report rate and an increase in packet collisions as the channel utilization increases. ETSI recommends that the channel load should not exceed $25 \%$ and congestion control methods are used to monitor the channel and reduce the CAM report rate if the local channel load becomes too high. As a leading vehicle by definition becomes a regular vehicle as soon as there is a vehicle in front within transmission range, the report rate will automatically return to default value (e.g. 2 $\mathrm{Hz}$ ) when road traffic increases, e.g. when the vehicle approaches an urban area or a densely trafficked road.

\section{SYSTEM MODEL}

To evaluate our approach a simulation study of the Overtaking Assistance application was conducted.

\section{The road traffic model:}

We consider a straight, single-lane, rural road with a speed limit of $90 \mathrm{~km} / \mathrm{h}(\sim 25 \mathrm{~m} / \mathrm{s})$. We further assume that all vehicles are equipped with 802.11 p-enabled communication technology and use a common and fixed output power that allows a transmission range of around $600 \mathrm{~m}$ at full LOS. A group of vehicles is introduced at the opposite ends of the test track. The distance between the vehicles in each group is $50 \mathrm{~m}$, which, for speeds around $90 \mathrm{~km} / \mathrm{h}$, is lower than the recommended "3 second rule", but likely more realistic. All vehicles within a group form a queue and maintain a fixed, randomly selected speed. Here, we are using a normal distribution with a mean of $90 \mathrm{~km} / \mathrm{h}$ and a standard deviation of 3 , covering the speed variations typically found on rural roads.

\section{The channel model:}

As we consider a straight single-lane road, we assume free LOS along the entire track. A simple channel model for this case was deduced from real-world measurement campaigns conducted with 802.11 p-equipped vehicles in various road settings [6]. Figure 2 shows the connectivity in a LOS environment between a (stationary) sending vehicle and a receiving vehicle moving at around $70 \mathrm{~km} / \mathrm{h}$. Our measurements indicate that a packet reception ratio close to 1 is kept up to a distance of about $400 \mathrm{~m}$ before it drops until connectivity is lost completely at a distance of about $600 \mathrm{~m}$. The packet reception probability $\left(P_{P R}\right)$ for leading vehicles is therefore modeled accordingly by fitting a curve to the results of the measurement campaign:

$$
\begin{aligned}
d \leq 400, & P_{P R}=0.999 \\
400<d \leq 500, & P_{P R}=\left(-\frac{2}{5} d+210\right) / 100 \\
500<d \leq 600, & P_{P R}=0.1 \\
d>600, & P_{P R}=0
\end{aligned}
$$

where $d$ denotes the distance between transmitting and receiving vehicle. For regular vehicles, $P_{P R}$ is reduced by a factor of 0.8 to account for the signal blocking effect of one or more vehicles situated between sender and receiver.

\section{The data traffic model:}

According to the ETSI standard, each vehicle sends out a CAM at a rate of $2 \mathrm{~Hz}$. We use a packet size of 800 byte and a data rate of $6 \mathrm{Mbit} / \mathrm{s}$ according to the standard. Furthermore, we use the priority scheme introduced in Section III and the report rate settings shown in Table 2 for leading and regular vehicles respectively.

TABLE 2: PRIORITY-BASED REPORT RATE

\begin{tabular}{|l|c|c|}
\hline & regular vehicle & leading vehicle \\
\hline Case 1 & $2 \mathrm{~Hz}$ & $2 \mathrm{~Hz}$ \\
\hline Case 2 & $2 \mathrm{~Hz}$ & $10 \mathrm{~Hz}$ \\
\hline Case 3 & $2 \mathrm{~Hz}$ & $20 \mathrm{~Hz}$ \\
\hline Case 4 & $10 \mathrm{~Hz}$ & $20 \mathrm{~Hz}$ \\
\hline
\end{tabular}

\section{SIMULATOR DETAILS}

The performance improvement of the Overtaking Assistance application when using our proposed prioritization scheme is studied through computer simulations in Matlab. The simulator implements all the functionalities of EDCA, but with the parameter settings introduced in Section IV. All vehicles are created simultaneously, with a common and fixed speed per driving direction. All vehicles generate CAMs periodically, starting with a randomized offset to prevent all vehicles that are created at the same time to start their periods simultaneously.

In our evaluation, we focus on the perspective of the leading vehicles, i.e., we are interested in when a leading vehicle for the first time becomes aware of any on-coming traffic by successfully receiving a CAM transmitted from the opposite driving direction. We consider two cases: 


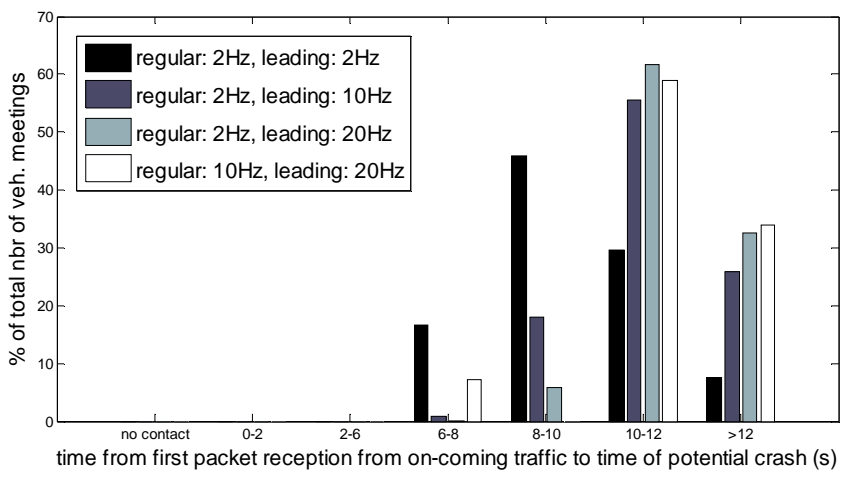

Figure 3: Reaction time from single side contact to potential crash. 2 vehicles per driving direction.

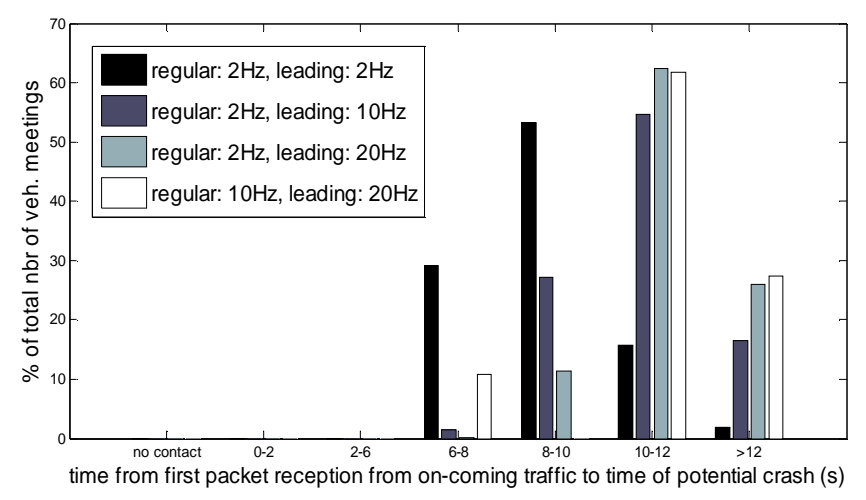

Figure 4: Reaction time from full contact to potential crash. 2 vehicles per driving direction.

- Single-sided contact: At least one driving direction has gained awareness of the on-coming vehicles.

- Full contact: Both sides have successfully received at least one CAM from a vehicle in the opposite driving direction and are aware of the hazardous traffic situation.

We would like to know how an increase in the CAM report rate influences the performance of the Overtaking Assistance application, i.e., is the driver reaction time increased or does a dramatic increase in the CAM report rate translate into congestion with negative consequences for this and other applications? As evaluation criteria we have therefore selected the following parameters:

- Reaction time: The time left for a driver to react to a warning, i.e., the time between the first successful packet reception and the potential crash if speeds are maintained.

- Distance: The distance between the position of a leading vehicle when single-sided contact is established and the position of the potential crash if speeds are maintained. For full contact, the leading vehicle with the shortest distance to the position of the potential crash is considered.

- Delay: The delay between the point in time when a leading vehicle (e.g. vehicle A in Figure 1) has entered into the transmission range of the vehicle in the opposite direction (vehicle B in Figure 1) and sent its first packet to the point in time of the first successful contact (a packet reception at $\mathrm{A}$ or $\mathrm{B})$.

\section{SIMULATION RESULTS}

In Figure 3 - 6, we can see the effect of the CAM report rate adaptation on the reaction time. Figure 3 shows the reaction

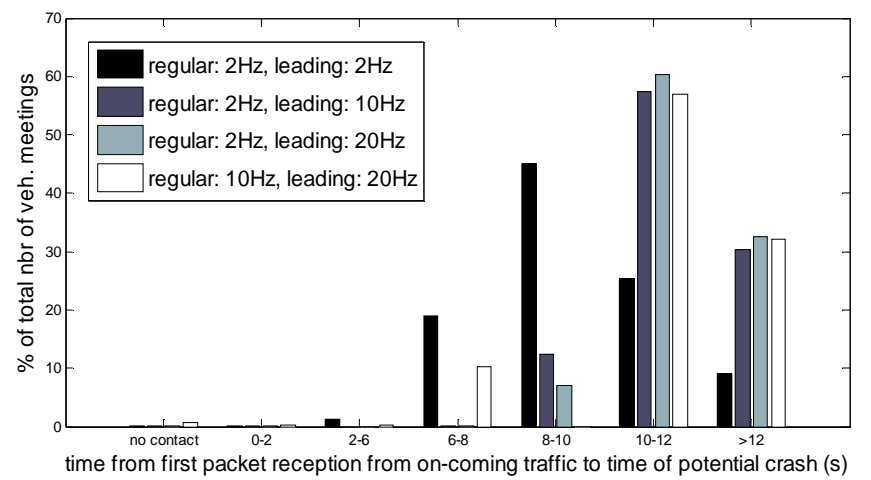

Figure 5 Reaction time from single side contact to potential crash. 10 vehicles per driving direction.

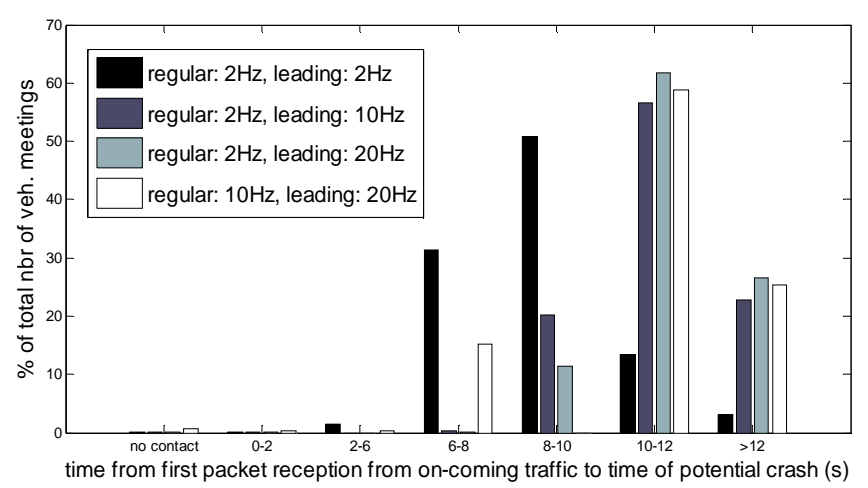

Figure 6: Reaction time from full contact to potential crash. 10 vehicles per driving direction.

time left when single-sided contact is established, while Figure 4 shows the reaction time left when full contact is established. In both cases, queues of two vehicles in each direction were simulated, whereas Figures 5 and 6 show singlesided and full contact for ten vehicles per driving direction. The presented simulation results are based on a series of 2000 vehicle meetings. For Priority Case 1 (a report rate of 2 $\mathrm{Hz}$ for regular and for leading vehicles) about $30 \%$ of the simulated vehicle meetings resulted in a reaction time of 6-8 s after both driving directions established contact (Figure 4). For Priority Cases 2 and 3 with increased report rate for leading vehicles a reaction time of at least $10 \mathrm{~s}$ is achieved in $70 \%$ and $90 \%$ of the cases, respectively. This can also been seen in Tables 3 and 4, which show the average Reaction time, Distance and Delay at Single-sided and Full contacts for simulations with queues containing two respective ten vehicles in each direction. It can be concluded that an increased report rate for leading vehicles improves the performance of an Overtaking Assistance application significantly. The delay until full contact has been established is cut in half by increasing the report rate of a leading vehicle from 2 to $10 \mathrm{~Hz}$ and reduced by $80 \%$ for an increase from 2 to $20 \mathrm{~Hz}$. The distance to the potential crash is increased by more than $100 \mathrm{~m}$ for all priority scenarios simply by increasing the report rate for leading vehicles from 2 to $20 \mathrm{~Hz}$. With about $2.5 \mathrm{~s}$ longer reaction time, the improvement is significant. Although, even without our prioritization scheme, a driver is provided with a seemingly comfortable reaction time of about $6 \mathrm{~s}$, one has to keep in mind that we simulate a straight road with full LOS. Under less ideal circumstances (e.g. over a crest) the point in time when on-coming traffic is 
detectable is considerably later and, therefore, the gain in driver reaction time through our prioritization scheme increases in significance.

For both the two and the ten vehicle scenario, Case 4 shows a slight decrease in performance compared to Case 3 . This is explained by the additional data traffic introduced by the regular nodes of Case 4 . Case 4 , where the report rate of the regular vehicles also is increased to $10 \mathrm{~Hz}$, has no positive effect as we only consider contact between the two driving directions and the leading vehicles still use a report rate of $20 \mathrm{~Hz}$ for both cases. This is also clear from Figures 5 and 6 which present results for ten vehicles per driving direction. All priority classes are affected by the overall increase in data traffic through the introduction of additional nodes in the network and for all classes there were a small percentage of the vehicle meetings where no contact could be established. Overall, Case 4 is affected most negatively by the additional data traffic produced by regular vehicles, which decreases the chances of the leading vehicles to get channel access.

TABLE 3: 2 VEHICLES

Average delay, distance and reaction time from 2000 vehicle meetings

\begin{tabular}{|l|c|c|c|c|c|c|}
\hline & \multicolumn{3}{|c|}{ Single-Side Contact } & \multicolumn{3}{c|}{ Full Contact } \\
\hline & Delay $(s)$ & Dist. $(m)$ & Time $(s)$ & Delay $(s)$ & Dist. $(m)$ & Time $(s)$ \\
\hline Case 1 & 2.41 & 474 & 9.58 & 3.17 & 437 & 8.82 \\
\hline Case 2 & 0.87 & 554 & 11.18 & 1.31 & 533 & 10.75 \\
\hline Case 3 & 0.45 & 578 & 11.60 & 0.68 & 564 & 11.35 \\
\hline Case 4 & 0.47 & 576 & 11.59 & 0.71 & 564 & 10.82 \\
\hline
\end{tabular}

TABLE 4: 10 VEHICLES

Average delay, distance and reaction time from 2000 vehicle meetings

\begin{tabular}{|c|c|c|c|c|c|c|}
\hline & \multicolumn{2}{|c|}{ Single-Side Contact } & \multicolumn{3}{c|}{ Full Contact } \\
\hline & Delay $(s)$ & Dist. $(\mathrm{m})$ & Time $(\mathrm{s})$ & Delay $(\mathrm{s})$ & Dist. $(\mathrm{m})$ & Time $(\mathrm{s})$ \\
\hline Case 1 & 2.60 & 468 & 9.45 & 3.35 & 431 & 8.70 \\
\hline Case 2 & 0.66 & 566 & 11.40 & 1.00 & 549 & 11.06 \\
\hline Case 3 & 0.47 & 575 & 11.57 & 0.72 & 563 & 11.13 \\
\hline Case 4 & 0.63 & 566 & 11.44 & 0.88 & 554 & 10.67 \\
\hline
\end{tabular}

Figure 7 shows the channel busy time as perceived by a leading vehicle, i.e. the percentage of time the channel is occupied by periodic CAM broadcasts. This number, which is referred to as the channel load within ETSI, is recommended to be kept below $25 \%$ in order to avoid channel congestion such that event triggered safety warnings are blocked. All four simulated report rate combinations for regular and leading vehicles are way below this threshold. Case $3(2 \mathrm{~Hz}$ report rate for regular and $20 \mathrm{~Hz}$ for leading vehicles), which was found to give the best results in our Overtaking Assistance application, only uses between $2.6 \%$ (for two vehicles per driving direction) and $3.9 \%$ (in case of ten vehicle per driving direction).

\section{CONCLUSIONS}

We have evaluated how IEEE 802.11p and cooperative awareness messaging between vehicles can be used as the

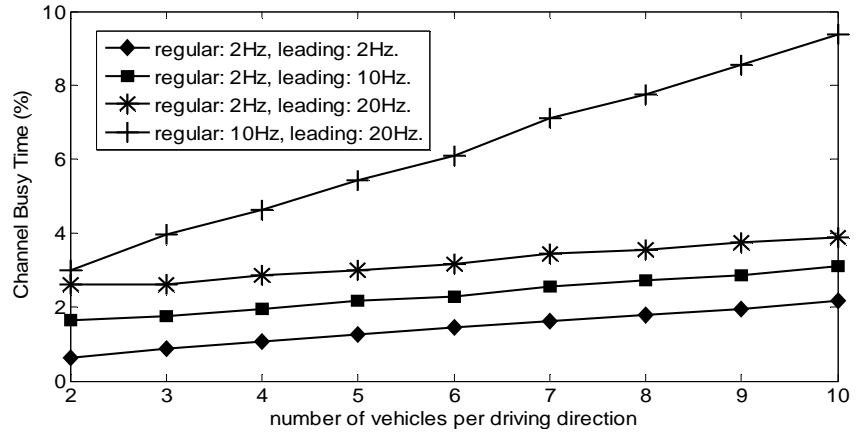

Figure 7: Channel Busy Time for varying numbers of vehicles and CAM report rates

basis for applications like overtaking assistance on sparsely trafficked rural roads. The limited number of communicating nodes on rural roads allows increasing the report rate of the cooperative awareness messages considerably, while still only occupying a relatively small share of the total available bandwidth. We therefore proposed a prioritization scheme based on adapting the report rate depending on which role the vehicle has in the application. A simulation study of the overtaking assistance application using a channel model derived from real-world vehicle-to-vehicle measurements shows that context-aware report rate adaptations lead to a significant reduction in response time. By that drivers are provided with valuable extra seconds to react to a safety warning and avert potential accidents on rural roads.

\section{REFERENCES}

[1] IEEE 802.11p Part11: Wireless LAN Medium Access Control (MAC) and Physical Layer (PHY) specifications: Amendment 7: Wireless Access in Vehicular Environment, July 2010.

[2] ETSI TC ITS, ES 202 663: European profile standard for the physical and medium access control layer of Intelligent Transport Systems operating in the $5 \mathrm{GHz}$ frequency band, 2009.

[3] ETSI TC ITS TS 102 637-2 Vehicular Communications; Basic Set of Applications; Part 2: Specification of Cooperative Awareness Basic Service, V1.1.1, 2010.

[4] ETSI TC ITS, TS 102 637-3 Vehicular Communications; Basic Set of Applications; Part 3: Specification of Decentralized Environmental Notification Basic Service, V1.1.1, 2010

[5] "Safety Strategies for Rural Roads", OECD Publishing, 1999. (summary: http://www.oecd.org/dataoecd/59/2/2351720.pdf).

[6] A. Böhm, K. Lidström, M. Jonsson and T. Larsson, "Evaluating CALM M5-based vehicle-to-vehicle communication in various road settings through field trials", Proc. IEEE LCN Workshop on User Mobility and Vehicular Networks, Denver, CO, USA, October 2010.

[7] A. Vinel, D. Staehle and A. Turlikov, "Study of beaconing for car-to-car communication in vehicular ad-hoc networks," Proc. IEEE Int. Conf. Communications, Dresden, Germany, June 2009, pp. 1-5.

[8] R. K. Schmidt et al., "Exploration of Adaptive Beaconing for Efficient Intervehicle Safety Communication", IEEE Network, vol. 24, no. 1, pp. 14-19, February 2010.

[9] M. Sepulcre, J. Gozalvez, J. Härri, and H. Hartenstein, "Application-Based Congestion Control Policy for the Communication Channel in VANETs," IEEE Communications Letters, vol. 14, no. 10, pp. 951-953, October 2010. 